\title{
GROUP INVERSE OF MODIFIED MATRICES OVER AN ARBITRARY RING*
}

\author{
N. CASTRO-GONZÁLEZ
}

\begin{abstract}
We focus on the group inverse of modified matrices $M=A-B C$, where $A$ is an $n \times n$ matrix with entries in an arbitrary ring $\mathcal{R}$ with unity and $B, n \times k$, and $C, k \times n$, are matrices having entries in $\mathcal{R}$. We assume that $A$ has the group inverse and we give conditions that guarantee the existence of the group inverse of $M$. We present an extension of the Sherman-Morrison-Woodbury formulae for the group inverse of $M$. Some particular cases and applications of the results obtained are discussed.
\end{abstract}

Key words. Group inverse, Generalized inverses, Sherman-Morrison-Woodbury formula.

AMS subject classifications. 15A09, 65F20, 65F 35 .

1. Introduction. Let $\mathcal{R}$ be a ring with identity 1 . We will denote by $\mathcal{R}^{n}$ the ring of all $n \times n$ matrices over $\mathcal{R}$ with identity $I_{n}$. Throughout this paper, $M$ is $A-B C$, where $A$ is an element of the ring $\mathcal{R}^{n}, B$ and $C$ stand for $n \times k$ and $k \times n$ matrices over $\mathcal{R}$. It is well known that if $A$ is invertible, then $M$ is invertible if and only if $S=I_{k}-C A^{-1} B$ is an invertible $k \times k$ matrix. Under the condition stated above, the Sherman-Morrison-Woodbury type formula holds:

$$
M^{-1}=\left(I_{n}+A^{-1} B S^{-1} C\right) A^{-1} .
$$

Applications of identities like this and its generalizations are indicated in [10,12, 21. Here we pay attention to the singular case and we replace inverses by generalized inverses. In the Markov chain context, the most relevant generalized inverse is the group inverse [13]. Formulae for the group inverse $M^{\sharp}$ of rank-one updates of a given complex matrix $A$ to the matrix $M=A+b c^{*}$, where $b$ and $c$ are $n \times 1$ complex matrices, were given by Meyer and Shoaf [15, Theorem 2.1]. Similar formulae for the Moore-Penrose inverse were derived by Meyer [14 and revisited by Baksalary et al. 11. For results on generalized inverses of modified matrices $A-B C$ under some restrictions we refer to [4, 9, 11, 16, 20,22].

We are interested in finding formulae for the group inverse of updated matrix in

${ }^{*}$ Received by the editors on July 3, 2012. Accepted for publication on March 3, 2013. Handling Editor: Oskar Maria Baksalary. The research is partly supported by Project MTM2010-18057, "Ministerio de Ciencia e Innovación" of Spain.

${ }^{\dagger}$ Facultad de Informática, Universidad Politécnica de Madrid, 28660 Boadilla del Monte, Madrid, Spain (nieves@fi.upm.es). 
the general case. We recall that a matrix $A^{\sharp} \in \mathcal{R}^{n}$ is the group inverse of $A \in \mathcal{R}^{n}$, if

$$
A A^{\sharp} A=A, \quad A^{\sharp} A A^{\sharp}=A^{\sharp}, \quad A A^{\sharp}=A^{\sharp} A .
$$

It is unique when it exists. Let $\mathcal{R}^{n, \sharp}$ be the set of all $A$ such that $A^{\sharp}$ exists. If $A$ is invertible, then $A^{\sharp}=A^{-1}$. We define $A^{\pi}$ to be $I_{n}-A A^{\sharp}$ for any $A \in \mathcal{R}^{n, \sharp}$. Then $A A^{\pi}=A^{\pi} A=0, A+A^{\pi}$ is invertible and $\left(A+A^{\pi}\right)^{-1}=A^{\sharp}+A^{\pi}$.

In our approach, we will deal with inner and reflexive inverses 2, 19]. An $m \times n$ matrix $A$ with entries in $\mathcal{R}$ is von Neumann regular, (briefly, regular) if there exists an $n \times m$ matrix $A^{-}$such that $A A^{-} A=A$. In this case, $A^{-}$is called an inner inverse or $\{1\}$-inverse of $A$. Let $A\{1\}$ denote the set of all $\{1\}$-inverses of $A$.

An $n \times m$ matrix $A^{+}$is called a reflexive inverse or $\{1,2\}$-inverse of $A$ if $A A^{+} A=$ $A$ and $A^{+} A A^{+}=A^{+}$. We observe that if $A$ is regular, then $A^{+}=A^{-} A A^{-}$is a $\{1,2\}$ inverse of $A$ for any $A^{-} \in A\{1\}$.

Next lemma gives an expression for inner inverses similar to (1.1).

Lemma 1.1. Let $A \in \mathcal{R}^{n}$ be invertible. We have that $M=A-B C$ is regular if and only if $S=I_{k}-C A^{-1} B$ is regular matrix of order $k$. In this case, a $\{1\}$-inverse of $M$ takes the form

$$
M^{-}=\left(I_{k}-A^{-1} B C\right)^{-} A^{-1}=A^{-1}+A^{-1} B S^{-} C A^{-1} .
$$

The result (1.2) is easy to verify. Puystjens-Hartwig [18, Theorem 9] and PatricioVeloso [17, Proposition 2.1] characterize the group invertibility of a regular element in terms of units.

Lemma 1.2. Let $A \in \mathcal{R}^{n}$ be regular. Then the group inverse of $A$ exists if and only if $U=A+I_{n}-A A^{-}$is invertible, independent of the choice of $A^{-}$or, equivalently, $W=A+I_{n}-A^{-} A$ is invertible, in which case, $A^{\sharp}=U^{-2} A=A W^{-2}$.

In Section 2, we will be concerned with the extension of the Sherman-MorrisonWoodbury formula for the group inverse of $A-B C$. We will present necessary and sufficient conditions for $(A-B C)^{\sharp}$ to exist, and when it exists, we will derive a formula for its computation. In Section 3, some particular cases of the results obtained are discussed and some numerical examples are given.

We conclude this section with a lemma which will be used in Section 2 .

Lemma 1.3. Let $A \in \mathcal{R}^{n}$ and let $F \in \mathcal{R}^{n}$ be idempotent such that $F A=F$. Then $(A-F)^{\sharp}$ exists if and only if $A^{\sharp}$ exists and $A^{\pi} F=0$. In this case,

$$
(A-F)^{\sharp}=A^{\sharp}-\left(A^{\sharp}\right)^{2} F .
$$


Proof. First, assume $A^{\sharp}$ exists and $A^{\pi} F=0$. We will prove that $X=A^{\sharp}-\left(A^{\sharp}\right)^{2} F$ satisfies the three required conditions in the definition of the group inverse of $A-F$. Since $F A=F$, it follows that $F=F A^{\sharp}$. This gives

$$
(A-F) X=X(A-F)=A A^{\sharp}-A^{\sharp} F
$$

and so the commutative property holds. Further,

$$
X(A-F) X=\left(A^{\sharp}-\left(A^{\sharp}\right)^{2} F\right)\left(A A^{\sharp}-A^{\sharp} F\right)=A^{\sharp}-\left(A^{\sharp}\right)^{2} F .
$$

As $F=A A^{\sharp} F$, we have

$$
(A-F) X(A-F)=\left(A A^{\sharp}-A^{\sharp} F\right)(A-F)=A-F .
$$

Consequently, $(A-F)^{\sharp}=A^{\sharp}-\left(A^{\sharp}\right)^{2} F$.

Conversely, assume $(A-F)^{\sharp}$ exists. It is fairly easy to see that the group inverse of $A$ exists and it is given by

$$
A^{\sharp}=(A-F)^{\sharp}(I-F)+(A-F)^{\pi} F \text {. }
$$

Hence, $A A^{\sharp} F=A(A-F)^{\pi} F=F(A-F)^{\pi} F$, and thus,

$$
A^{\pi} F=F-F(A-F)^{\pi} F=F(A-F)(A-F)^{\sharp} F=0 .
$$

This completes the proof.

Next, we state the analogue of the above lemma.

Lemma 1.4. Let $A \in \mathcal{R}^{n}$ and let $F \in \mathcal{R}^{n}$ be idempotent such that $A F=F$. Then $(A-F)^{\sharp}$ exists if and only if $A^{\sharp}$ exists and $F A^{\pi}=0$. In this case,

$$
(A-F)^{\sharp}=A^{\sharp}-F\left(A^{\sharp}\right)^{2} \text {. }
$$

REMARK 1.5. Let $A$ and $F$ be $n \times n$ complex matrices and $r=\operatorname{rank} F, 0<r<n$. If $F$ is idempotent, then $F=U^{-1}\left[\begin{array}{ll}I & 0 \\ 0 & 0\end{array}\right] U$. Write $A=U^{-1}\left[\begin{array}{ll}A_{11} & A_{12} \\ A_{21} & A_{22}\end{array}\right] U$ as block matrices conformal with $F$. From condition $F A=F$ it follows that $A=$ $U^{-1}\left[\begin{array}{cc}I & 0 \\ A_{21} & A_{22}\end{array}\right] U$. The eigenvalues of $A$ are the eigenvalues of $A_{22}$ together with $r$ ones. From [13, Lemma 2.1], it follows that $A^{\sharp}$ exists if and only if $A_{22}^{\sharp}$ exists. In this case, $A^{\sharp}=U^{-1}\left[\begin{array}{cc}I & 0 \\ X & A_{22}^{\sharp}\end{array}\right] U$ with $X=-A_{22}^{\sharp} A_{21}+A_{22}^{\pi} A_{21}$ and $A^{\pi}=$ 
$U^{-1}\left[\begin{array}{cc}0 & 0 \\ -A_{22}^{\pi} A_{21} & A_{22}^{\pi}\end{array}\right] U$, where $A_{22}^{\pi}=I-A_{22} A_{22}^{\sharp}$. Since $A-F=U^{-1}\left[\begin{array}{cc}0 & 0 \\ A_{21} & A_{22}\end{array}\right] U$, the eigenvalues of $A-F$ are the eigenvalues of $A_{22}$ together with $r$ zeros. Consequently, if $A$ has the eigenvalue $\lambda=0$ with multiplicity $s$, then $A-F$ has the eigenvalue $\lambda=0$ with multiplicity $r+s$. By [13, Lemma 2.1], we conclude that $(A-F)^{\sharp}$ exists if and only if the group inverse of $A_{22}$ exists and $A_{22}^{\pi} A_{21}=0$. So, both the existence of $A^{\sharp}$ and condition $A^{\pi} F=0$ are needed to guarantee the existence of the group inverse of $A-F$.

2. Group inverses of modified matrices. This section gives a characterization of the existence of the group inverse of modified matrices $M=A-B C$ in terms of $k \times k$ invertible matrices over $\mathcal{R}$. We give a formula that updates $A^{\sharp}$ to $M^{\sharp}$, which is an extension of the Sherman-Morrison-Woodbury updating formula. First, we consider the particular case where $A$ is invertible.

Theorem 2.1. Let $A \in \mathcal{R}^{n}$ be invertible and let $S=I_{k}-C A^{-1} B$ be regular. Set $T=I_{k}-S S^{-}$for a fixed but arbitrary $S^{-}$. Then the group inverse of $M$ exists if and only if $V=S+T C A^{-2} B$ is invertible in $\mathcal{R}^{k}$, in which case

$$
M^{\sharp}=\left(I_{n}+A^{-1} B V^{-1} C-Z\right) A^{-1}\left(I_{n}-Z\right) \quad \text { and } \quad M^{\pi}=Z,
$$

where $Z=A^{-1} B V^{-1} T C A^{-1}$.

Proof. Since $S$ is a regular element of $\mathcal{R}^{k}$, by Lemma 1.1 $M=A-B C$ is a regular element of $\mathcal{R}^{n}$. Now, $M^{\sharp}$ exists if and only if $U=M+I_{n}-M M^{-}$is invertible, independent of the choice of $M^{-}$, by Lemma 1.2 In this case,

$$
M^{\sharp}=U^{-2} M \text {. }
$$

Taking a $M^{-}$which is of the form (1.2), we obtain

$$
U=A-B\left(C-T C A^{-1}\right),
$$

where $T=I_{k}-S S^{-}$for a fixed but arbitrary $S^{-}$. Hence, $U$ is invertible in $\mathcal{R}^{n}$ if and only if $V=I-\left(C-T C A^{-1}\right) A^{-1} B$ is invertible in $\mathcal{R}^{k}$. Moreover, applying (1.1) yields

$$
U^{-1}=\left(I_{n}+A^{-1} B V^{-1}\left(C-T C A^{-1}\right)\right) A^{-1} .
$$

Hence,

$$
U^{-1} M=I_{n}-A^{-1} B V^{-1} T C A^{-1} .
$$

Substituting (2.3) and (2.4) into (2.2) we conclude that first formula in (2.1) holds. The second formula in (2.1) follows immediately from the first. 
If, in addition, $S$ is invertible, then with $S^{-}=S^{-1}$ (2.1) becomes (1.1).

We can state an analogue of Theorem 2.1, replacing in the proof above the requirement that $U=M+I-M M^{-}$is invertible by the equivalent condition that $W=M+I_{n}-M^{-} M$ is invertible, and using $M^{\sharp}=A W^{-2}$.

Theorem 2.2. Let $A \in \mathcal{R}^{n}$ be invertible and let $S=I_{k}-C A^{-1} B$ be regular. Set $\hat{T}=I_{k}-S^{-} S$ for a fixed but arbitrary $S^{-}$. Then the group inverse of $M$ exists if and only if $\hat{V}=S+C A^{-2} B \hat{T}$ is invertible in $\mathcal{R}^{k}$, in which case

$$
M^{\sharp}=\left(I_{n}-\hat{Z}\right) A^{-1}\left(I_{n}+B \hat{V}^{-1} C A^{-1}-\hat{Z}\right) \quad \text { and } \quad M^{\pi}=\hat{Z},
$$

where $\hat{Z}=A^{-1} B \hat{T} \hat{V}^{-1} C A^{-1}$.

We emphasize that apart from $A^{-1}$, expressions (2.1) and (2.5) involve inverses and inner inverses of order $k$. Consequently, these formulae have computational advantage whenever $k$ is considerably less than $n$.

We can now formulate our main result.

Theorem 2.3. Let $A \in \mathcal{R}^{n, \sharp}$ and let $A^{\pi} B$ be regular. Set $Q=I_{k}-\left(A^{\pi} B\right)^{+} A^{\pi} B$, $S=\left(I_{k}-C A^{\sharp} B\right) Q-C A^{\pi} B$, and $T=I_{k}-S S^{-}$for a fixed but arbitrary $\left(A^{\pi} B\right)^{+}$ and $S^{-}$. If $S$ is regular, then $M^{\sharp}$ exists if and only if

$$
V=S+T\left(I_{k}-C A^{\sharp}\left(B-A^{\sharp} B Q\right)\right) \text { is invertible in } \mathcal{R}^{k} \quad \text { and } \quad B T C A^{\pi}=0 .
$$

In this case,

$$
M^{\sharp}=\left(I_{n}+\alpha V^{-1}\left(C-T C A^{\sharp}\right)\right) A^{\sharp}\left(I_{n}-\sigma V^{-1} \delta\right)-\alpha V^{-1}\left(I_{k}-Q\right) V^{-1} \delta
$$

and

$$
M^{\pi}=A^{\pi}+\alpha V^{-1} \delta
$$

where

$$
\alpha=A^{\sharp} B Q+A^{\pi} B, \quad \delta=T C A^{\sharp}+C A^{\pi}, \quad \sigma=A^{\sharp} B Q-B\left(I_{k}-Q\right) .
$$

Proof. Throughout the proof, set $F=A^{\pi}-A^{\pi} B\left(A^{\pi} B\right)^{+} A^{\pi}$ and $R=A-B C+F$. We note that $F$ is idempotent. We have that $F B=F A=0$, and thus, $F R=F$. By Lemma 1.3.

$$
(R-F)^{\sharp} \text { exists if and only if } R^{\sharp} \text { exists and } R^{\pi} F=0,
$$

in which case

$$
M^{\sharp}=(R-F)^{\sharp}=R^{\sharp}-\left(R^{\sharp}\right)^{2} F,
$$


and hence,

$$
M^{\pi}=R^{\pi}+R^{\sharp} F .
$$

Define $G=\left(A+A^{\pi}\right)\left(I_{n}+A^{\sharp} B\left(A^{\pi} B\right)^{+} A^{\pi}\right)$ and $D=C+\left(A^{\pi} B\right)^{+} A^{\pi}$. We can rewrite

$$
R=G-B D
$$

We observe that $A^{\sharp} B\left(A^{\pi} B\right)^{+} A^{\pi}$ is 2-nilpotent, and thus, $I_{n}+A^{\sharp} B\left(A^{\pi} B\right)^{+} A^{\pi}$ is invertible. Consequently, $G$ is also invertible and $G^{-1}=\left(I_{n}-A^{\sharp} B\left(A^{\pi} B\right)^{+} A^{\pi}\right)\left(A^{\sharp}+\right.$ $\left.A^{\pi}\right)$. Now, let $S=I_{k}-D G^{-1} B$. An easy computation shows that

$$
S=\left(I_{k}-C A^{\sharp} B\right) Q-C A^{\pi} B .
$$

Since $S$ is regular, it follows from Theorem 2.1 that

$(G-B D)^{\sharp}$ exists if and only if $V=S+T D G^{-2} B$ is invertible in $\mathcal{R}^{k}$.

In this case,

$$
R^{\sharp}=\left(I_{n}+G^{-1} B V^{-1} D-Z\right) G^{-1}\left(I_{n}-Z\right) \quad \text { and } \quad R^{\pi}=Z,
$$

where $Z=G^{-1} B V^{-1} T D G^{-1}$.

Now, we proceed to show that condition $R^{\pi} F=0$ is equivalent to $B T C A^{\pi}=0$. We check that

$$
G^{-1} F=F, \quad S\left(A^{\pi} B\right)^{+} A^{\pi}=C\left(F-A^{\pi}\right), \quad T D F=T C F=T C A^{\pi} .
$$

On account of the above relations, we have $Z F=0$ if and only if $B V^{-1} T C A^{\pi}=0$, which turns out to be equivalent to $B T C A^{\pi}=0$ because $B V=U B$, where $U=$ $I_{n}-B\left(D G^{-1}-T D G^{-2}\right)$ is invertible in $\mathcal{R}^{n}$ whenever $V$ is invertible in $\mathcal{R}^{k}$.

Assuming $Z F=0$ to hold, we conclude from (2.11) that

$$
R^{\sharp} F=\left(I_{n}+G^{-1} B V^{-1} C\right) F .
$$

It is easily seen that $D G^{-2} B=I-C A^{\sharp} B+C\left(A^{\sharp}\right)^{2} B Q-S$. Thus,

$$
V=S+T D G^{-2} B=S+T\left(I_{k}-C A^{\sharp}\left(B-A^{\sharp} B Q\right)\right),
$$

and hence,

$$
\begin{aligned}
Z A^{\pi} & =G^{-1} B V^{-1} T\left(I_{k}-C A^{\sharp} B\right)\left(A^{\pi} B\right)^{+} A^{\pi} \\
& =G^{-1} B V^{-1}(V-S)\left(A^{\pi} B\right)^{+} A^{\pi} \\
& =\left(I_{n}+G^{-1} B V^{-1} C\right)\left(A^{\pi}-F\right),
\end{aligned}
$$


the last equality being a consequence of the second relation in (2.12) and the fact that $G^{-1} B\left(A^{\pi} B\right)^{+} A^{\pi}=A^{\pi} B\left(A^{\pi} B\right)^{+} A^{\pi}=A^{\pi}-F$. On the other hand, using $G^{-1} A A^{\sharp}=A^{\sharp}$ and $D G^{-1} A A^{\sharp}=C A^{\sharp}$, we obtain

$$
Z A A^{\sharp}=G^{-1} B V^{-1} T C A^{\sharp} .
$$

Substituting (2.11), (2.13), (2.15) and (2.14) into (2.9), we obtain

$$
M^{\sharp}=\left(I_{n}+G^{-1} B V^{-1} D-Z\right) G^{-1}\left(A A^{\sharp}-G^{-1} B V^{-1}\left(T C A^{\sharp}+C A^{\pi}\right)\right) .
$$

With the notation (2.8), we have that

$$
G^{-1} B=\alpha, \quad G^{-2} B=A^{\sharp} \sigma+A^{\pi} B,
$$

and thus, (2.16) becomes

$$
\begin{aligned}
M^{\sharp} & =\left(I_{n}+\alpha V^{-1}\left(C+\left(A^{\pi} B\right)^{+} A^{\pi}\right)-Z\right)\left(A^{\sharp}-\left(A^{\sharp} \sigma+A^{\pi} B\right) V^{-1} \delta\right) \\
& =\left(I_{n}+\alpha V^{-1}\left(C-T C A^{\sharp}\right)\right) A^{\sharp}\left(I_{n}-\sigma V^{-1} \delta\right)-\Sigma V^{-1} \delta,
\end{aligned}
$$

where

$$
\Sigma=\left(I_{n}+\alpha V^{-1}\left(C+\left(A^{\pi} B\right)^{+} A^{\pi}\right)-Z\right) A^{\pi} B=\alpha V^{-1}\left(I_{k}-Q\right),
$$

the last equality being a consequence of (2.14). This establishes formula (2.6). In the same manner we can see that (2.10) becomes

$$
M^{\pi}=Z+R^{\sharp} F=A^{\pi}+\alpha V^{-1} \delta,
$$

and (2.7) is proved. $\square$

If $A$ is invertible, then $A^{\sharp}=A^{-1}, A^{\pi}=0$, and (2.6) becomes (2.1).

We can now state the analogue of Theorem 2.2, which can be proved, using Lemma 1.4 and Theorem 2.3, in much the same way as previous theorem.

Theorem 2.4. Let $A \in \mathcal{R}^{n, \sharp}$ and let $C A^{\pi}$ be regular. Set $\hat{Q}=I_{n}-\left(C A^{\pi}\right)^{+} C A^{\pi}$, $\hat{S}=\hat{Q}\left(I_{k}-C A^{\sharp} B\right)-C A^{\pi} B$, and $\hat{T}=I-\hat{S}^{-} \hat{S}$ for a fixed but arbitrary $\left(C A^{\pi}\right)^{+}$and $\hat{S}^{-}$. If $\hat{S}$ is regular then $M^{\sharp}$ exists if and only if

$$
\hat{V}=\hat{S}+\left(I_{k}-\left(C-\hat{Q} C A^{\sharp}\right) A^{\sharp} B\right) \hat{T} \quad \text { is invertible in } \mathcal{R}^{k} \quad \text { and } \quad A^{\pi} B \hat{T} C=0 .
$$

In this case,

$$
M^{\sharp}=\left(I_{n}-\hat{\delta} \hat{V}^{-1} \hat{\sigma}\right) A^{\sharp}\left(I_{n}+\left(B-A^{\sharp} B \hat{T}\right) \hat{V}^{-1} \hat{\alpha}\right)-\hat{\delta} \hat{V}^{-1}\left(I_{k}-\hat{Q}\right) \hat{V}^{-1} \hat{\alpha}
$$

and

$$
M^{\pi}=A^{\pi}+\hat{\delta} \hat{V}^{-1} \hat{\alpha}
$$


where

$$
\hat{\alpha}=\hat{Q} C A^{\sharp}+C A^{\pi}, \quad \hat{\delta}=A^{\sharp} B \hat{T}+A^{\pi} B, \quad \hat{\sigma}=\hat{Q} C A^{\sharp}-\left(I_{k}-\hat{Q}\right) C .
$$

3. Consequences and examples. In this section, we formulate some important consequences of the theorems.

Theorem 2.3 specializes to the following result if $A^{\pi} B=0$.

Corollary 3.1. Let $A \in \mathcal{R}^{n, \sharp}$. Assume $A^{\pi} B=0$ and let $S=I_{k}-C A^{\sharp} B$ be regular. Set $T=I-S S^{-}$for a fixed but arbitrary $S^{-}$. Then $M^{\sharp}$ exists if and only if

$$
V=S+T C\left(A^{\sharp}\right)^{2} B \text { is invertible in } \mathcal{R}^{k} \quad \text { and } \quad B T C A^{\pi}=0 .
$$

In this case,

$$
M^{\sharp}=\left(I_{n}+A^{\sharp} B V^{-1}\left(C-T C A^{\sharp}\right)\right) A^{\sharp}\left(I_{n}-A^{\sharp} B V^{-1} \delta\right)
$$

and $M^{\pi}=A^{\pi}+A^{\sharp} B V^{-1} \delta$, where $\delta=T C A^{\sharp}+C A^{\pi}$.

Proof. In the notation of Theorem 2.3, we have $Q=I_{k}, S$ and $V$ as in the statement of the corollary. Moreover, $\alpha$ and $\sigma$ defined as in (2.8) become $\alpha=\sigma=$ $A^{\sharp} B$. Hence, the corollary follows from Theorem 2.3.

If in addition either $S$ is invertible or $S=0$, then (3.1) gives reduced expressions for $M^{\sharp}$. For the first case, our formula agrees with the one given in [8] Theorem 4.1].

Corollary 3.2. Let $A \in \mathcal{R}^{n, \sharp}, A^{\pi} B=0$, and let $S=I_{k}-C A^{\sharp} B$ be invertible in $\mathcal{R}_{k}$. Then $M^{\sharp}$ exists and

$$
M^{\sharp}=\left(I_{n}+A^{\sharp} B S^{-1} C\right) A^{\sharp}\left(I_{n}-A^{\sharp} B S^{-1} C A^{\pi}\right) .
$$

Corollary 3.3. Let $A \in \mathcal{R}^{n, \sharp}, A^{\pi} B=0$, and let $C A^{\sharp} B=I_{k}$. Then $M^{\sharp}$ exists if and only if $V=C\left(A^{\sharp}\right)^{2} B$ is invertible in $\mathcal{R}_{k}$ and $C A^{\pi}=0$, in which case

$$
M^{\sharp}=\left(I_{n}+A^{\sharp} B V^{-1} C\left(I_{n}-A^{\sharp}\right)\right) A^{\sharp}\left(I_{n}-A^{\sharp} B V^{-1} C A^{\sharp}\right) .
$$

Recall that an $n \times k$ matrix $B$ is said to have a left inverse if there is a $k \times n$ matrix $X$ such that $X B=I_{k}$. If $B_{L}^{-1}$ is a left inverse of $B$, then $B$ is regular and $B^{+}=B_{L}^{-1}$ is a $\{1,2\}$-inverse of $B$. Theorem 2.3 specializes to the following result if $A^{\pi} B$ has a left inverse.

Corollary 3.4. Let $A \in \mathcal{R}^{n, \sharp}$ and assume that $A^{\pi} B$ has a left inverse. Set $S=-C A^{\pi} B$ and $T=I-S S^{-}$for a fixed but arbitrary $S^{-}$. If $S$ is regular then $M^{\sharp}$ 
exists if and only if

$$
V=S+T\left(I_{k}-C A^{\sharp} B\right) \text { is invertible in } \mathcal{R}^{k} \quad \text { and } \quad B T C A^{\pi}=0 .
$$

In this case,

$$
M^{\sharp}=\left(I_{n}+A^{\pi} B V^{-1}\left(C-T C A^{\sharp}\right)\right) A^{\sharp}\left(I_{n}+B V^{-1} \delta\right)-A^{\pi} B V^{-2} \delta,
$$

and $M^{\pi}=A^{\pi}+A^{\pi} B V^{-1} \delta$, where $\delta=T C A^{\sharp}+C A^{\pi}$.

Proof. Choose $\left(A^{\pi} B\right)^{+}=\left(A^{\pi} B\right)_{L}^{-1}$. In the notation of Theorem 2.3], we have $Q=I_{k}-\left(A^{\pi} B\right)_{L}^{-1} A^{\pi} B=0, S$ and $V$ as in the statement of the corollary. Moreover, $\alpha$ and $\sigma$ defined as in (2.8) become $\alpha=A^{\pi} B$ and $\sigma=-B$. Therefore the corollary follows from Theorem 2.3, $\square$

If in addition either $S$ invertible or $S=0$, then (3.4) takes a simpler form.

Corollary 3.5. Let $A \in \mathcal{R}^{n, \sharp}$ and assume that $A^{\pi} B$ has a left inverse. If $S=-C A^{\pi} B$ is invertible, then $M^{\sharp}$ exists, in which case

$$
M^{\sharp}=\left(I_{n}+A^{\pi} B S^{-1} C\right) A^{\sharp}\left(I_{n}+B S^{-1} C A^{\pi}\right)-A^{\pi} B S^{-2} C A^{\pi} .
$$

Corollary 3.6. Let $A \in \mathcal{R}^{n, \sharp}$ and assume that $A^{\pi} B$ has a left inverse. If $C A^{\pi} B=0$ then $M^{\sharp}$ exists if and only if

$$
V=I_{k}-C A^{\sharp} B \quad \text { is invertible in } \mathcal{R}^{k} \quad \text { and } \quad B C A^{\pi}=0 .
$$

In this case,

$$
M^{\sharp}=\left(I_{n}-A^{\pi} B V^{-1} C A^{\sharp}\right) A^{\sharp}\left(I_{n}+B V^{-1} C A^{\sharp}\right) .
$$

Let us mention that if $A$ is an $n \times n$ matrix over a field $\mathcal{R}$ such that $A$ has group inverse, $b$ is of order $n \times 1$ over $\mathcal{R}$ and $c$ is of order $1 \times n$ over $\mathcal{R}$, then for the update of the group inverse of $A-b c$, we have to distinguish four cases. Indeed, we have that $A^{\pi} b$ either equals the zero matrix or it has full column rank equal to 1 . In this latter case, we know that $A^{\pi} b$ is regular and any $\{1\}$-inverse of $A^{\pi} b$ is a left inverse. Moreover, since $s=\left(1-c A^{\sharp} b\right) Q-c A^{\pi} b$ is an element of the field $\mathcal{R}$, it follows that either $s$ has an inverse or $s=0$. Hence, $(A-b c)^{\sharp}$, when it exists, can be computed using one of the forms (3.2), (3.3), (3.5) and (3.6). Consequently, Corollaries 3.2 and 3.3 and Corollaries 3.5 and 3.6 coincide with the results given in [15. Theorem 2.1] if $A-b c$ is a rank-one update of a complex matrix. 
Similarly, from Theorem 2.4 we can derive some special cases. We pay attention to the case $C A^{\pi}=0$.

Corollary 3.7. Let $A \in \mathcal{R}^{n, \sharp}$ and assume that $C A^{\pi}=0$. Set $\hat{S}=I_{k}-C A^{\sharp} B$, and $\hat{T}=I-\hat{S}^{-} \hat{S}$ for a fixed but arbitrary $\hat{S}^{-}$. If $\hat{S}$ is regular then $M^{\sharp}$ exists if and only if

$$
\hat{V}=\hat{S}+C\left(A^{\sharp}\right)^{2} B \hat{T} \quad \text { is invertible in } \mathcal{R}^{k} \quad \text { and } \quad A^{\pi} B \hat{T} C=0 \text {. }
$$

In this case,

$$
M^{\sharp}=\left(I_{n}-\hat{\delta} \hat{V}^{-1} C A^{\sharp}\right) A^{\sharp}\left(I_{n}+\left(B-A^{\sharp} B \hat{T}\right) \hat{V}^{-1} C A^{\sharp}\right)
$$

and

$$
M^{\pi}=A^{\pi}+\hat{\delta} \hat{V}^{-1} C A^{\sharp},
$$

where $\hat{\delta}=A^{\sharp} B \hat{T}+A^{\pi} B$.

Next, we show that Corollary 3.1 and Corollary 3.7 agree when we assume both conditions $A^{\pi} B=0$ and $C A^{\pi}=0$.

Corollary 3.8. Let $A \in \mathcal{R}^{n, \sharp}, A^{\pi} B=0, C A^{\pi}=0$, and let $S=I_{k}-C A^{\sharp} B$ be regular. Set $T=I_{k}-S S^{-}$and $\hat{T}=I_{k}-S^{-} S$ for a fixed but arbitrary $S^{-}$. Then $M^{\sharp}$ exists if and only if $V=S+T C\left(A^{\sharp}\right)^{2} B$ is invertible in $\mathcal{R}_{k}$ or, equivalently, $\hat{V}=S+C\left(A^{\sharp}\right)^{2} B \hat{T}$ is invertible. In this case,

$$
\begin{aligned}
M^{\sharp} & =\left(I_{n}+A^{\sharp} B V^{-1}\left(C-T C A^{\sharp}\right)\right) A^{\sharp}\left(I_{n}-A^{\sharp} B V^{-1} T C A^{\sharp}\right) \\
& =\left(I_{n}-A^{\sharp} B \hat{T} \hat{V}^{-1} C A^{\sharp}\right) A^{\sharp}\left(I_{n}+\left(B-A^{\sharp} B \hat{T}\right) \hat{V}^{-1} C A^{\sharp}\right) .
\end{aligned}
$$

One more case merits mentioning here.

Corollary 3.9. Let $A$ be idempotent such that $A B=B$. Let $S=I_{k}-C A B$ be regular. Then $M^{\sharp}$ exists if and only if $S^{\sharp}$ exists and $B S^{\pi} C(I-A)=0$, in which case

$$
M^{\sharp}=A-A B S^{\sharp} C A-A B\left(S^{\sharp}\right)^{2} C(I-A)-A B S^{\pi} C .
$$

Proof. Since $A$ is idempotent, $A$ has the group inverse, $A^{\sharp}=A$ and $A^{\pi}=I_{n}-A$. Condition $A B=B$ implies $A^{\pi} B=0$, and thus, we can apply Corollary 3.1. We have that $V=S+T C\left(A^{\sharp}\right)^{2} B=S+T C A B=S+T(I-S)=S+T$, where $T=I_{k}-S S^{-}$. Thus, $V$ is invertible if and only if $S^{\sharp}$ exists, by Lemma 1.2. Consequently, $M^{\sharp}$ exists if and only if $S^{\sharp}$ exists and $B S^{\pi} C(I-A)=0$, taking $S^{-}=S^{\sharp}$. Now, replacing $T$ by 
$I_{k}-S S^{\sharp}$ and using that $V^{-1}=\left(S+I_{k}-S S^{\sharp}\right)^{-1}=S^{\sharp}+I_{k}-S S^{\sharp}$, it follows from (3.1) that

$$
\begin{aligned}
M^{\sharp} & =\left(I_{n}+A B V^{-1}(C-T C A)\right) A\left(I_{n}-A B V^{-1}(T C A+C(I-A))\right) \\
& =\left(A+A B S^{\sharp} C A\right)\left(I-A B\left(S^{\sharp}+I_{k}-S S^{\sharp}\right)\left(C+S S^{\sharp} C A\right)\right) \\
& =A-A B S^{\sharp} C A-A B\left(S^{\sharp}\right)^{2} C(I-A)-A B S^{\pi} C,
\end{aligned}
$$

which establishes (3.7).

A simple case can be derived from previous result, putting $A=I_{n}$, 3. Theorem 3.5.].

EXAMPLE 3.10. Let $\mathbb{Z}_{12}$ be the ring of integers modulo 12. Let $A=\left[\begin{array}{lll}1 & 1 & 0 \\ 0 & 0 & 0 \\ 0 & 0 & 1\end{array}\right]$ over $\mathbb{Z}_{12}$. We observe that $A$ is idempotent and thus has the group inverse, and $A^{\sharp}=$ $A$. Let $B=\left[\begin{array}{l}3 \\ 0 \\ 1\end{array}\right]$ and $C=\left[\begin{array}{lll}1 & 4 & 1\end{array}\right]$. Consider $M=A-B C=\left[\begin{array}{ccc}10 & 1 & 9 \\ 0 & 0 & 0 \\ 11 & 8 & 0\end{array}\right]$. We obtain $A^{\pi}=I_{3}-A=\left[\begin{array}{ccc}0 & 11 & 0 \\ 0 & 1 & 0 \\ 0 & 0 & 0\end{array}\right]$. Hence, $A^{\pi} B=0$. Let $S=1-C A^{\sharp} B=1-4=9$.

Then $S$ is a group invertible element of $\mathbb{Z}_{12}, S^{\sharp}=9$, and $S^{\pi}=1-S S^{\sharp}=1-9=4$. We have $S^{\pi} C A^{\pi}=4\left[\begin{array}{lll}0 & 3 & 0\end{array}\right]=\left[\begin{array}{lll}0 & 0 & 0\end{array}\right]$. Thus, by previous result $M$ has the group inverse. We use (3.7) to compute $M^{\sharp}=\left[\begin{array}{lll}4 & 7 & 3 \\ 0 & 0 & 0 \\ 5 & 2 & 6\end{array}\right]$.

Let $M$ be the modification of matrix $A=\left[a_{i j}\right]_{1 \leq i, j \leq n}$ to the form $M=\left[\widetilde{a}_{i j}\right]_{1 \leq i, j \leq n}$ where $\widetilde{a}_{i j}=a_{i j}$ for $i=k+1, \ldots, n$ (only the first $k$ rows are modified). We can write

$$
M=A-\left[\begin{array}{c}
I_{k} \\
0_{(n-k) \times k}
\end{array}\right]\left[\begin{array}{ccc}
a_{11}-\widetilde{a}_{11} & \cdots & a_{1 n}-\widetilde{a}_{1 n} \\
\vdots & & \vdots \\
a_{k 1}-\widetilde{a}_{k 1} & \cdots & a_{k n}-\widetilde{a}_{k n}
\end{array}\right]:=A-B C .
$$

Note that $A^{\pi} B$ is the submatrix of $A^{\pi}$ from column 1 to $k$, which will be denoted by $A_{k}^{\pi}$. Once we have computed the group inverse of $A$ and a $\{1,2\}$-inverse of $A_{k}^{\pi}$ we can use them for successively updating the group inverse of $A$. Each time the first $k$ rows are modified, we obtain the $k \times k$ matrices $S$ and $V$ defined as in the statement of Theorem 2.3 and we compute an inner inverse of $S$ and the inverse of $V$ to produce 
$M^{\sharp}$ using formula (2.6).

$$
\begin{aligned}
\text { EXAMPLE 3.11. Let } A= & {\left[\begin{array}{cccc}
0 & -1 & 0 & 1 \\
-1 & -1 & 0 & 0 \\
1 & -1 & 1 & 0 \\
1 & 1 & 0 & 0
\end{array}\right], B=\left[\begin{array}{ll}
1 & 0 \\
0 & 1 \\
0 & 0 \\
0 & 0
\end{array}\right] \text {, and } } \\
C & =\left[\begin{array}{cccc}
-1 & 0 & 1 & 1 \\
1 & -1 & 1 & 0
\end{array}\right] .
\end{aligned}
$$

We modify the two first rows of $A$ to produce $M=A-B C=\left[\begin{array}{cccc}1 & -1 & -1 & 0 \\ -2 & 0 & -1 & 0 \\ 1 & -1 & 1 & 0 \\ 1 & 1 & 0 & 0\end{array}\right]$.

We have

$$
A^{\sharp}=\left[\begin{array}{cccc}
1 / 2 & -1 / 4 & 0 & 3 / 4 \\
-1 / 2 & -1 / 4 & 0 & -1 / 4 \\
-1 & -1 & 1 & -2 \\
1 / 2 & 1 / 4 & 0 & 1 / 4
\end{array}\right], \quad A^{\pi} B=\left[\begin{array}{cc}
0 & -1 / 2 \\
0 & 1 / 2 \\
0 & 1 \\
0 & 1 / 2
\end{array}\right] .
$$

We take the $\{1,2\}$-inverse of $A^{\pi} B,\left(A^{\pi} B\right)^{+}=\left[\begin{array}{cccc}0 & 0 & 0 & 0 \\ -2 & 0 & 0 & 0\end{array}\right]$. Set $Q=I_{2}-$ $\left(A^{\pi} B\right)^{+} A^{\pi} B$.

Further, $S=\left(I_{2}-C A^{\sharp} B\right) Q-C A^{\pi} B=\left[\begin{array}{cc}2 & -2 \\ 0 & 0\end{array}\right]$. We choose $S^{-}=\left[\begin{array}{cc}1 / 2 & 0 \\ 0 & 0\end{array}\right]$, and set $T=I_{2}-S S^{-}$. Then

$$
V=S+T\left(I_{2}-C A^{\sharp}\left(B-A^{\sharp} B Q\right)\right)=\left[\begin{array}{cc}
2 & -2 \\
-1 & 2
\end{array}\right] .
$$

We have that $V$ is nonsingular and $B T C A^{\pi}=0$, and thus, the group inverse of $A-B C$ exists. We obtain

$$
(A-B C)^{\sharp}=\left[\begin{array}{cccc}
1 / 4 & -1 / 2 & -1 / 4 & 0 \\
-1 / 4 & -1 / 2 & -3 / 4 & 0 \\
-1 / 2 & 0 & 1 / 2 & 0 \\
3 / 4 & 1 / 2 & 1 / 4 & 0
\end{array}\right],
$$


applying formula (2.6).

In this paper, we have focussed on the extension of the Sherman-MorrisonWoodbury formula for the group inverse of $A-B C$. Our next purpose is to establish analogous results for the Moore-Penrose. This topic will be developed in a forthcoming paper.

Acknowledgment. The author gratefully acknowledges the comments and suggestions of the anonymous reviewers and associate editor O.M. Baksalary that have improved the presentation of this paper.

\section{REFERENCES}

[1] J.K. Baksalary, O.M. Baksalary, and G. Trenkler. A revisitation of formulae for the MoorePenrose inverse of modified matrices. Linear Algebra Appl., 372:207-224, 2003.

[2] A. Ben-Israel and T.N.E. Greville. Generalized Inverses. Theory and Applications, second edition. Springer-Verlag, New York, 2003.

[3] N. Castro-González, C. Mendes-Arajo, and P. Patricio. Generalized inverses of a sum in rings. Bull. Aust. Math. Soc., 82:156-164, 2010.

[4] N. Castro-González and M.F. Martínez-Serrano. Expressions for the g-Drazin inverse of additive perturbed elements in a Banach algebra. Linear Algebra Appl., 432:1885-1895, 2010.

[5] D.S. Cvetković-Illić. Expression of the Drazin inverse and MP-inverse of partitioned matrix and quotient identity of generalized Schur complement. Appl. Math. Comput., 213:18-24, 2009.

[6] D.S. Cvetković-Illić and Y. Wei. Representations for the Drazin inverse of bounded operators on Banach space. Electron J. Linear Algebra, 18:613-627, 2009.

[7] C.Y. Deng. On the invertibility of the operator A-XB. Numer. Linear Algebra Appl., 16:817-831, 2009.

[8] E. Dopazo and M.F. Martínez-Serrano. On deriving the Drazin inverse of a modified matrix. Doi:10.1016/j.laa.2011.06.023, 2011.

[9] J.A. Fill and D.E. Fishkind. The Moore-Penrose generalized inverse fo sums of matrices. SIAM J. Matrix Anal. Appl., 21(2):53-60, 2000.

[10] M. Fiedler. Remarks on the Sherman-Morrison-Woodbury formulae. Mathematica Bohemica, 3:253-262, 2003.

[11] R.E. Hartwig, G. Wang, and Y. Wei. Some additive results on the Drazin inverse. Linear Algebra Appl., 322:207-217, 2001.

[12] H.V. Henderson and S.R. Searle. On deriving the inverse of a sum of matrices. SIAM Rev., 23(1):125-139, 1982.

[13] C.D. Meyer. The role of the group generalized inverse in the theory of finite Markov chains. SIAM Rev., 17:443-464, 1975.

[14] C.D. Meyer. Generalized inversion of modified matrices. SIAM J. Appl. Math., 24:315-323, 1973.

[15] C.D. Meyer and J.M. Shoaf. Updating finite Markov chains by using techniques of group matrix inversion. J. Statist. Comput. Simulation, 11:163-181, 1980.

[16] P. Patricio and R. Puystjens. Generalized invertiblility in two semigroups of a ring. Linear Algebra Appl., 377:53-60, 2004.

[17] P. Patricio and A. Veloso da Costa On the Drazin index of regular elements. Cent. Eur. J. Math., 7(2):200-208, 2009. 
[18] R. Puystjens and R.E. Hartwig. The group of a companion matrix. Linear Multilinear Algebra, 43:137-150, 1997.

[19] C.R. Rao and S.K. Mitra. Generalized inverse of a matrix and its applications. Proc. Sixth Berkeley Symp. on Math. Statist. and Prob., Univ. of Calif. Press, Vol. 1, 601-620, 1972.

[20] K.S. Riedel. A Sherman-Morrison-Woodbury identity for rank augmenting matrices with applications to centering. SIAM J. Matrix Anal. Appl., 13(2):659-662, 1992.

[21] T. Steerneman and F. van Perlo-ten Kleij. Properties of the matrix $A-X Y^{*}$. Linear Algebra Appl., 410:53-60, 2005.

[22] Y. Wei. The Drazin inverse of a modified matrix. Appl. Math. Comput., 125:295-301, 2002. 